\title{
PARTICIPAČ̃̃O DE ADOLESCENTES BRASILEIROS NAS AULAS DE EDUCAÇÃO FÍSICA ESCOLAR: REVISÃO SISTEMÁTICA
}

\author{
Bárbara Gicélia da Silva Araújo \\ Universidade Federal de Pernambuco, Recife, Pernambuco, Brasil. \\ Rafael Miranda Tassitano \\ Universidade Federal Rural de Pernambuco, Recife, Pernambuco, Brasil. \\ Mallu Dias \\ Universidade Estadual de Pernambuco, Recife, Pernambuco, Brasil. \\ Maria Cecília Marinho Tenório \\ Universidade Federal Rural de Pernambuco, Recife, Pernambuco, Brasil.
}

\begin{abstract}
Resumo
Identificar e comparar artigos sobre a participação de adolescentes brasileiros nas aulas de Educação Física (EF) e verificar os fatores associados constituem os objetivos deste trabalho. O método utilizado foi busca sistematizada da literatura nas bases: MEDLINE, Scielo, LILACS, Google acadêmico, ERIC, CAPES e Adolec. Disso, dez estudos foram incluídos, sendo $70 \%$ realizados no Nordeste e $100 \%$ utilizaram questionário. A participação variou de 29,3 a 75,0\%. A variável comportamental associada à participação mais avaliada foi a prática de atividade física. Portanto, conclui-se que poucos estudos foram realizados, sendo a maioria deles no Nordeste. Dado isso, este trabalho incentiva a produção de estudos em outras regiões do Brasil, a utilização de outros instrumentos, a investigação dos fatores associados e a identificação dos motivos que levam à participação nas aulas de EF.
\end{abstract}

Palavras-chave: Estudantes. Atividade física. Educação física. Brasil.

\section{PARTICIPATION OF BRAZILIAN ADOLESCENTS IN SCHOOL PHYSICAL EDU- CATION CLASSES: SYSTEMATIC REVIEW}

\begin{abstract}
Objective: To identify and compare articles on physical education participation of Brazilian adolescents and associated factors. Methods: A systematic search was conducted using with these databases: MEDLINE, SciELO, LILACS, Google academic, ERIC, CAPES and Adolec. Results: Ten studies were included. $70 \%$ were conducted in the Northeast, $100 \%$ used a questionnaire. The participation range for 29.3 to $75.0 \%$. The behavioral variable associated with participation was physical activity. Conclusion: Few studies have been carried out. All used a questionnaire and a majority was conducted in the Northeast. Production is encouraged in other regions of Brazil, using other instruments, investigation of the associated factors and identification of the reasons that lead to participation in EF classes.
\end{abstract}

Keywords: Students. Physical activity. Physical education. Brazil. 


\title{
PARTICIPACIÓN DE ADOLESCENTES BRASILEÑOS EN LAS CLASES DE EDU- CACIÓN FÍSICA ESCOLAR: REVISIÓN SISTEMÁTICA
}

\begin{abstract}
Resumen
Objetivo: Identificar y comparar las publicaciones sobre participación en las clases de Educación Física y sus factores asociados en adolescentes brasileños. Métodos: Búsqueda sistemática de la literatura en las bases: MEDLINE, SciELO, LILACS, Google académico, ERIC, CAPES y Adolec. Resultados: Diez estudios fueron incluidos. La mayoría realizada en el Nordeste. La participación en las clases de EF varió de 29,3 a 75,0\%. La variable comportamental asociada a la participación fue la práctica de actividad física. Conclusión: Pocos estudios realizados. La mayoría realizados en el Nordeste. Se fomenta la producción de estudios en otras regiones de Brasil, utilización de otros instrumentos, investigación de los factores asociados e identificación de los motivos que conducen a la participación en las clases de EF.
\end{abstract} Palabras clave: Estudiantes. Actividad física. Educación física. Brasil.

\section{Introdução}

A Educação Física (EF) escolar proporciona inúmeros benefícios aos estudantes, como a saúde física, cognitiva (JANSSEN; LEBLANC, 2010; REZENDE et al., 2015) e social (LAZZOLI et al., 1998; SANTOS et al., 2015). No entanto, nota-se que entre os adolescentes, principalmente as meninas, a proporção de não participação nas aulas de EF está elevada e os motivos ainda não estão definidos na literatura (COLEDAM et al., 2014; KREMER, REICHERT; HALLAL, 2012). Neto et al. (2010), ao investigarem o desinteresse dos alunos em participar da aula de EF no Ensino Médio, notaram que tal fato ocorria principalmente durante a transição do Ensino Fundamental para o Médio, e essa ocorrência pode estar relacionada a experiências anteriores vivenciadas pelos estudantes. Contudo, não foram investigados quais os principais fatores relacionados a essa falta de interesse pelos alunos.

Além dos benefícios retrocitados, a prática da EF na escola favorece a autonomia dos alunos para monitorar as próprias atividades, regulando o esforço, traçando metas, conhecendo potencialidades, limitações e sabendo distinguir situações de trabalho corporal que podem ser prejudiciais (BRASIL, 1997). Do mesmo modo, deveria ofertar aos adolescentes partes significativas da quantidade adequada de atividade física (AF) recomendada pela OMS (2014), que é 60 minutos de intensidade moderada a vigorosa todos os dias. No entanto, alguns estudos mostram que há muitos adolescentes que não atendem às recomendações de AF diária, apresentando níveis elevados de inatividade física (HALLAL et al., 2010; BARUFALDI et al., 2012). Além disso, estudos apontam para a diminuição nos níveis de aptidão aeróbia dos adolescentes no Brasil (SILVA et al., 2015), aumento na prevalência de excesso de peso (BENEDET et al., 2013; PINHO; BOTELHO; CALDEIRA, 2014), elevado volume de comportamento sedentário associado com níveis elevados de peso corporal e baixos níveis de AF (GUERRA; JUNIOR; FLORINDO, 2016) e elevada prevalência de sonolência diurna (SOUZA et al., 2007). As causas desses comportamentos de risco adotados por essa população ainda não estão bem descritas na literatura.

De acordo com as recomendações da Sociedade Brasileira de Medicina do Esporte, o incentivo à prática de $\mathrm{AF}$ na infância e na adolescência deve ser considerado como prioridade em nossa sociedade. A EF escolar realizada de forma adequada deve ser considerada essencial e parte indissociável do processo global de educação da população infantojuvenil (LAZZOLI et al., 1998). A identificação de fatores associados à participação nas aulas de EF, que podem ser sociais, emocionais, comportamentais e motivacionais, é um procedimento importante para conhecer o interesse dessa população e orientar o planejamento de programas de 
intervenção adequados para o Ensino Médio. O objetivo desta revisão sistemática foi identificar e comparar os resultados das publicações sobre a participação de adolescentes brasileiros nas aulas de EF e seus fatores associados.

\section{Métodos}

Foi realizada uma busca sistematizada da literatura no período de março de 2007 a março de 2017, nas seguintes bases de dados eletrônicas: MEDLINE, Scielo, LILACS, Google acadêmico, ERIC, CAPES (Catálogo de Teses e Dissertações) e Adolec. Foi realizada, adicionalmente, uma pesquisa nas referências dos artigos encontrados, com o propósito de identificar possíveis estudos que não haviam sido identificados na pesquisa inicial.

Os descritores usados foram previamente selecionados através de consulta aos DeCs (Ciências da Saúde, BIREME) e Mesh (Medical Subject Headings, Pubmed). Foram utilizados blocos de três conceitos distintos: o primeiro, com os termos relacionados à EF escolar ("Educação Física", "aulas", "atividade física" "atividade motora", "exercício físico"), o segundo, com termos relacionados à adolescência ("adolescente", "jovem", "juventude", "estudantes") e o terceiro, com termos relacionados ao País ("Brasil", "brasileiro (a)"). Foram considerados os estudos com amostras nacionais e foi utilizado o operador lógico "OR" para combinar os descritores dentro de cada bloco, bem como o "AND NOT" e o operador lógico "AND" para combinar os blocos. A pesquisa foi conduzida em português e inglês. Para ampliar a busca relacionada a artigos escritos em inglês, foram utilizados os truncamentos necessários. Foram utilizadas também as recomendações do protocolo MOOSE (Guidelines for Meta-Analyses and Systematic Reviews of Observational Studies), específico para estudos observacionais.

Foram adotados os seguites critérios de inclusão: (a) a adolescentes, (b) artigo original com seres humanos, (c) mencionar a participação nas aulas de Educação Física e (d) coleta de dados ter sido realizada no Brasil. Artigos de revisão não foram incluídos, assim como os estudos que analisavam a mesma população, sendo selecionado apenas um documento para representar a população estudada. Os artigos foram selecionados por dois revisores, a priori, por seu título e resumo. Em seguida, os artigos pré-selecionados foram lidos na íntegra por ambos os revisores. Em caso de discordância quanto à inclusão dos artigos, um terceiro revisor foi consultado. Os resultados foram tabulados no Microsoft Office Excel 2010, em computador pessoal, sob a responsabilidade e o gerenciamento da primeira autora. Além da participação nas aulas de EF, foram registradas informações sobre ano de publicação, Estado (região), tipo de escola (privada ou pública), instrumento de coleta, faixa etária (anos), amostra e os fatores associados à participação nas aulas de EF.

\section{Resultados}

O fluxograma do processo de seleção das publicações é apresentado na Figura 1. Dos 553 estudos identificados inicialmente (após a remoção de duplicatas), dez foram considerados elegíveis para esta revisão sistemática: nove artigos originais e uma tese. 
Figura 1. Fluxograma do processo de seleção das publicações

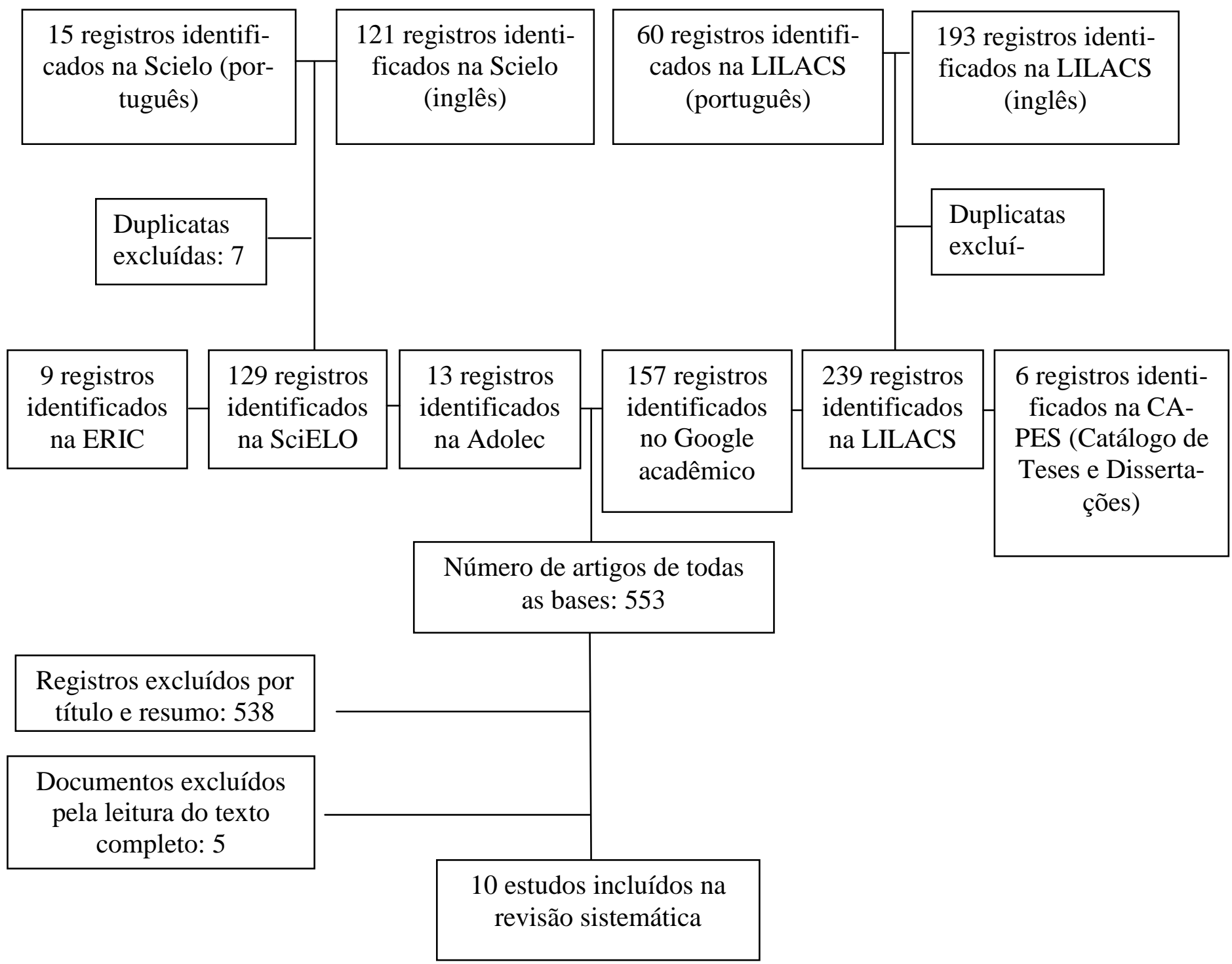

Fonte: Os autores (2019).

Dos 10 artigos incluídos, oito tinham como principal objetivo analisar a participação nas aulas de EF entre adolescentes. Outros dois artigos tinham objetivos primários diferentes, embora a participação na EF escolar fosse uma covariável dos estudos. Todos os artigos incluídos nesta revisão tinham delineamento transversal.

As características dos estudos estão apresentadas na Tabela 1. Seis artigos foram realizados na região Nordeste, seguidos das regiões Sudeste e Sul (dois artigos). Não se encontrou nenhum estudo realizado nas regiões Norte e Centro-Oeste do país. Dos 10 estudos analisados, seis foram realizados com amostra superior a 2.000 indivíduos. Em todos os estudos, a participação na EF escolar foi avaliada indiretamente, ou seja, com base nas informações fornecidas pelos indivíduos (questionários). Cinco estudos utilizaram questionários previamente validados, GSHS (Global School-Based Student Health Survey) e o COMPAC (Comportamentos de Adolescentes Catarinenses). 
Tabela 1. Características dos estudos sobre participação na Educação Física escolar por adolescentes brasileiros

\begin{tabular}{|c|c|c|c|c|c|c|}
\hline Primeiro Autor & $\begin{array}{l}\text { Ano de } \\
\text { publicação }\end{array}$ & $\begin{array}{l}\text { Estado } \\
\text { (região) }\end{array}$ & $\begin{array}{l}\text { Tipo de } \\
\text { escola }\end{array}$ & Instrumento & $\begin{array}{l}\text { Faixa } \\
\text { etária } \\
\text { (anos) }\end{array}$ & $\mathbf{N}$ \\
\hline Ceschini & 2009 & $\begin{array}{l}\text { São Paulo } \\
\text { (SE) }\end{array}$ & Pública & Questionário & 14 a 19 & 3.845 \\
\hline Silva & 2009 & $\begin{array}{l}\text { Santa Catari- } \\
\text { na } \\
(\mathrm{S})\end{array}$ & Pública & COMPAC & 15 a 19 & 5.028 \\
\hline Tassitano & 2010 & $\begin{array}{l}\text { Pernambuco } \\
\text { (NE) }\end{array}$ & Pública & GSHS & 14 a 19 & 4.210 \\
\hline Feitosa & 2011 & $\begin{array}{l}\text { Pernambuco } \\
\text { (NE) }\end{array}$ & Pública & COMPAC & 14 a 29 & 600 \\
\hline Paula & 2011 & $\begin{array}{l}\text { Minas Gerais } \\
(\mathrm{SE})\end{array}$ & & Questionário & 15 a 18 & 19 \\
\hline $\begin{array}{l}\text { Farias } \\
\text { Júnior }\end{array}$ & 2012 & $\begin{array}{l}\text { Paraíba } \\
(\mathrm{NE})\end{array}$ & $\begin{array}{l}\text { Públicas } \\
\text { e Priva- } \\
\text { das }\end{array}$ & Questionário & 14 a 19 & 2.874 \\
\hline Coledam & 2014 & $\begin{array}{l}\text { Paraná } \\
\text { (S) }\end{array}$ & Pública & Questionário & 10 a 20 & 827 \\
\hline Oliveira & 2014 & $\begin{array}{l}\text { Bahia } \\
\text { (NE) }\end{array}$ & Pública & Questionário & 11 a 24 & 86 \\
\hline Bezerra & 2015 & $\begin{array}{l}\text { Pernambuco } \\
\text { (NE) }\end{array}$ & Pública & GSHS & 14 a 19 & 6.264 \\
\hline Silva & 2016 & $\begin{array}{l}\text { Sergipe } \\
\text { (NE) }\end{array}$ & Pública & GSHS & 14 a 19 & 3.992 \\
\hline
\end{tabular}

Fonte: Os autores (2019).

A maioria dos estudos utilizou a pergunta: "Você participa das aulas de Educação Física?”, cuja Resposta: Sim ou Não. A Tabela 2 apresenta os valores percentuais de participação nas aulas de EF escolar, bem como os fatores associados (sociais, econômicos, demográficos e comportamentais). O percentual de participação nas aulas de EF variou de 29,3 a $75,0 \%$. Dentre os fatores associados, os fatores sociodemográficos foram os mais investigados e a variável comportamental foi a prática de atividade física.

Dos 10 estudos incluídos, apenas sete estratificaram a participação nas aulas de EF por gênero. Cinco identificaram percentuais de participação superiores entre os rapazes, quando comparados às moças: $78,2 \%$ versus $21,8 \%$ (CESCHINI 2009); 78,4\% versus $65,7 \%$ (COLEDAM, 2014); 69,2\% versus 65,5\% (FARIAS JÚNIOR et al., 2012); 39,5\% versus $32.2 \%$ (TASSITANO et al., 2010) e 79,2\% versus 72.5\% (SILVA E SMITH MENEZES, 2016). Porém, em dois foi observado o contrário, maior percentual de participação entre as moças: $39,5 \%$ versus $34,6 \%$ (FEITOSA et al, 201) e 53,9\% versus 49,0\% (SILVA et al., 2009). Dois artigos (PAULA; SILVA; KOCIAN, 2011; BEZERRA, 2015) não apresentaram distribuição estratificada por gênero. O estudo de Oliveira, Macedo e Silva (2014) foi realizado exclusivamente com adolescentes do gênero feminino.

Apenas dois estudos investigaram os motivos da participação na EF escolar. De acordo com Oliveira, Macedo e Silva (2014), a maioria dos estudantes investigados não participava das aulas porque não gostava de esportes, e o fator que levaria à participação nas 
aulas seria a diversificação das mesmas. Já o estudo de Paula, Silva e Kocian (2011) aponta como maior causa de evasão nas aulas de EF a falta de conteúdo, pois o professor deixa a aula livre, na maioria das vezes, influenciando negativamente o interesse do aluno.

Tabela 2. Percentuais de Participação de adolescentes brasileiros na Educação Física escolar e fatores associados

\begin{tabular}{|c|c|c|}
\hline $\begin{array}{c}\text { Primeiro autor, } \\
\text { ano }\end{array}$ & $\begin{array}{l}\text { Participação } \\
\text { nas aulas de } \\
\text { EF }(\%)\end{array}$ & Fatores associados à participação na EF escolar \\
\hline Bezerra, 2015 & 71,7 & $\begin{array}{l}\text { Sexo, mesorregião geográfica, zona de moradia, idade, } \\
\text { estado civil, situação ocupacional, possui computador, } \\
\text { escolaridade materna, turno escolar, série do curso, } \\
\text { comportamento sedentário. }\end{array}$ \\
\hline Ceschini, 2009 & 49,5 & $\begin{array}{l}\text { Gênero; idade; nível socioeconômico; região geográfi- } \\
\text { ca; conhece o Agita SP; uso de tabaco; consumo de } \\
\text { bebidas alcoólicas e tempo diário de TV (h/dia). }\end{array}$ \\
\hline Coledam, 2014 & 72,9 & $\begin{array}{l}\text { Sexo; idade; com quem mora; número de amigos no } \\
\text { bairro; condição socioeconômica; escolaridade materna } \\
\text { e paterna; prática de AF; percepção de AF; transporte } \\
\text { ativo; prática esportiva; comportamento sedentário; } \\
\text { necessidade de movimentação durante as aulas de EF; } \\
\text { local de aula de EF; prática de esportes com amigos do } \\
\text { bairro; local adequado para prática esportiva no bairro; } \\
\text { quantas vezes por semana utiliza os lugares para práti- } \\
\text { ca esportiva; caso não tenha disponibilidade no bairro, } \\
\text { qual lugar utiliza para prática esportiva. }\end{array}$ \\
\hline $\begin{array}{l}\text { Farias Júnior, } \\
2012\end{array}$ & 66,9 & $\begin{array}{l}\text { Sexo; idade; cor da pele; situação ocupacional (traba- } \\
\text { lho); condição econômica; escolaridade do pai e da } \\
\text { mãe; TV - meio de semana e fim de semana; computa- } \\
\text { dor/videogame - meio de semana e fim de semana; } \\
\text { estado nutricional; percepção de saúde. }\end{array}$ \\
\hline Feitosa, 2011 & 29,3 & $\begin{array}{l}\text { Sexo; faixa etária; filho; local de residência; mora com } \\
\text { a família; trabalho; renda familiar mensal; turno; série } \\
\text { escolar; a escola oferece aulas. }\end{array}$ \\
\hline Oliveira, 2014 & 47,8 & $\begin{array}{l}\text { Motivo da não participação nas aulas; incentivo a par- } \\
\text { ticipar das aulas; finalidade das aulas de Educação } \\
\text { Física. }\end{array}$ \\
\hline Paula, 2011 & -- & $\begin{array}{l}\text { Motivo da participação nas aulas; motivo da não parti- } \\
\text { cipação nas aulas; atividades quando não participa das } \\
\text { aulas; importância das aulas; conteúdos trabalhados } \\
\text { nas aulas; conteúdos preferidos nas aulas. }\end{array}$ \\
\hline Silva, 2009 & 45,7 & $\begin{array}{l}\text { Sexo; idade; renda familiar mensal; trabalha atualmen- } \\
\text { te; satisfação com o peso corporal; percepção de saúde; } \\
\text { percepção de cansaço no trabalho; com quem mora; } \\
\text { número de pessoas na residência; número de irmãos; } \\
\text { localidade em que reside; período em que estuda; des- } \\
\text { locamento da residência à escola e ao trabalho; fre- } \\
\text { quência de consumo diário de frutas e verduras; hábito } \\
\text { de fumar pelo menos uma vez por semana durante uma } \\
\text { semana típica; consumo de bebidas alcoólicas; experi- } \\
\text { ência com drogas ilícitas; prática de AF; comportamen- } \\
\text { to sedentário; turno de estudo. }\end{array}$ \\
\hline
\end{tabular}




\begin{tabular}{lll}
\hline Silva , 2016 & 75,0 & $\begin{array}{l}\text { Atividade física no tempo livre; atividades de lazer } \\
\text { fisicamente ativas. }\end{array}$ \\
\hline Tassitano, 2010 & 35,1 & $\begin{array}{l}\text { Prática de AF; assistir TV durante dias e fins de sema- } \\
\text { na; consumo de frutas; consumo de verduras; consumo } \\
\text { de álcool; consumo de refrigerante; fumar cigarros. }\end{array}$ \\
\hline
\end{tabular}

Fonte: Os autores (2019).

\section{Discussão}

Foram encontrados poucos artigos publicados sobre a participação nas aulas de EF e em metade deles o percentual de participação foi baixo $(\leq 50 \%)$. Foram encontrados outros artigos abordando a variável de interesse desta revisão, no entanto, investigavam a mesma população, sendo estes descartados nessa análise.

Apesar do baixo número de estudos, o tamanho e o processo de seleção da amostra demonstram a abrangência dos artigos incluídos. Observamos que a maioria possuía amostra maior que 2000 estudantes, e adotaram o processo de seleção probabilístico e por conglomerados, o que também expressa a representatividade da amostra dos artigos incluídos.

Observamos que a maioria dos estudos foi realizada na região Nordeste. Contudo, as diferenças culturais, econômicas e sociais entre os estudantes e as escolas das regiões do Brasil podem estar relacionadas à participação dos estudantes. Assim, é importante conhecer e comparar os resultados de outras regiões.

Todos utilizaram questionários para avaliação da participação, porém cinco não mencionaram informações sobre a validade deles. A importância da utilização de instrumentos validados está relacionada à capacidade de um instrumento medir o que realmente se deseja medir, além de visar melhorar a qualidade dos dados coletados e permitir comparações mais adequadas entre os estudos (REICHENHEIM; MORAES, 1998). Além disso, os questionários autorrelatados possuem limitações, como o viés de memória, por exemplo. Recomenda-se o uso de uma medida direta, principalmente quando se trata de avaliação de um comportamento. Neste caso, a participação nas aulas pode ser avaliada por meio de observação como também a utilização conjunta de questionários, pedômetros e/ou acelerômetros. Outro aspecto que pode contribuir na qualidade dos dados é a utilização conjunta da abordagem qualitativa. A utilização de grupo focal, por exemplo, pode auxiliar na avaliação do contexto das aulas, como também no conhecimento dos motivos para participação.

A maioria dos artigos observou uma baixa participação dos adolescentes nas aulas de EF escolar, corroborando estudos realizados em outros países. Na California Diamant, Babey e Wolstein (2011), ao investigarem a participação nas aulas de EF, observaram que a porcentagem de adolescentes participantes das aulas cai abruptamente com a idade, de 95\% de participação aos 12 anos para apenas $23 \%$ aos 17 anos. Uma justificativa plausível para essa ocorrência é que muitos alunos da escola obtêm isenções da EF nos últimos dois anos escolar. A participação nas aulas de EF é uma importante estratégia para aumentar o nível de atividade física dos adolescentes. Além disso, a participação pode estar associada à realização de atividades físicas fora da escola (DAUENHAUER; KEATING, 2011). Palakshappa et al. (2015), por exemplo, ao analisarem longitudinalmente a saúde de adolescentes norteamericanos, tomaram como preditor para a AF adulta a participação na EF escolar. Apesar do reconhecimento de tantos benefícios, a participação dos estudantes do Ensino Médio é baixa e os motivos ainda são inconsistentes.

O percentual de participação na EF escolar foi maior entre os rapazes, resultado semelhante aos achados em estudos internacionais (DIAMANT; BABEY; WOLSTEIN, 2011; CASTEJÓN; GIMÉNEZ, 2015). Essa diferença de gênero em relação à participação nas aulas de EF pode estar relacionada a alguns fatores, como culturais e comportamentais. Os estudos 
de Salles-Costa et al. (2003) e Uchoga e Altmann (2016) demonstraram que os rapazes parecem confiar mais nas suas habilidades esportivas do que as moças, assim arriscam-se mais nas aulas e, consequentemente, são mais participativos. Quanto aos fatores motivacionais, a moças geralmente valorizam os motivos relacionados com a saúde, enquanto os rapazes apresentam motivos relacionados com a competição (NUNES; PEREIRA; ESPADA, 2015).

O conteúdo programático também está relacionado a tal diferença de gênero na participação das aulas de EF escolar. Na maioria das aulas, o conteúdo selecionado estava relacionado aos esportes de preferência masculina, como o futebol, por exemplo. Dois estudos investigaram os motivos da participação na EF escolar. Em ambos os estudos, a falta de diversificação dos conteúdos das aulas de $\mathrm{EF}$ foi um fator diretamente relacionado ao interesse do aluno em participar das aulas. Os professores precisam conhecer as preferências dos alunos, especialmente entre os alunos do ensino médio, quanto aos conteúdos de suas aulas. Além disso, devem adequar esses conteúdos por gênero e elaborar estratégias de inclusão.

Outro fator associado à participação está relacionado à intensidade das aulas. Os estudos de Trost et al. (2002) e Kremer, Reichert e Hallal (2012) relatam a baixa participação de moças em atividades de intensidade vigorosa. Em algumas escolas, as aulas incluem atividades físicas de intensidade vigorosa, que geralmente não atraem o público feminino.

Quanto aos fatores associados à participação nas aulas de EF, foi observado que a prática de AF foi a variável mais investigada. Dos quatro estudos que avaliaram essa variável, dois (COLEDAM et al., 2014; SILVA et al., 2009) não observaram associação significativa entre participação na EF escolar e nível de atividade física. No entanto, Silva e SmithMenezes (2016) e Tassitano et al. (2010) observaram que os estudantes que participavam das aulas de EF apresentavam melhores atitudes quanto à prática de AF. Esses resultados podem ser explicados pela grande variabilidade das aulas, especialmente em relação à quantidade (dias por semana) e à duração (minutos por dia). É importante aprofundar o conhecimento sobre as aulas de EF e seus fatores associados. Dauenhauer e Keating (2011), ao investigarem o papel da EF na formação de padrões de AF, observaram que a EF pode ser uma fonte importante de AF, especialmente entre as meninas, e pode influenciar a participação em AF fora da sala de aula.

\section{Conclusão}

Nesta revisão, foram encontrados poucos estudos (10 publicações) que investigaram a participação nas aulas de EF. O percentual de participação nas aulas variou de 29,3 a 75,0\%. A maioria usou questionário e foi realizado na região Nordeste. Desse modo, incentiva-se a produção de mais estudos com diferentes instrumentos de medida e abordagens. Sugere-se também a investigação de outros fatores associados e identificação dos motivos que levam à participação nas aulas de EF.

\section{Referências}

BARUFALDI, L. A.; ABREU, G. A.; COUTINHO, E. S. F.; BLOCH, K. V. Meta-analysis of the prevalence of physical inactivity among Brazilian adolescents. Cad. Saúde Pública. Rio de Janeiro, v. 28, n. 6, p. 1019-1032, 2012.

BENEDET, J.; ASSIS, M. A. A.; CALVO, M. C. M.; ANDRADE, D. F. Excesso de peso em adolescentes: explorando potenciais fatores de risco. Revista Paulista de Pediatria. São Pulo, v. 31, n. 2, p. 172-181, 2013. 
BEZERRA, J. Atividade física e comportamento sedentário em adolescentes do estado de Pernambuco, Brasil: estudo comparativo de dois inquéritos (2006 e 2011). 2015. Tese (Doutorado) - Universidade Federal de Santa Catarina, Florianópolis, 2015.

BRASIL. Secretaria de Educação Fundamental. Parâmetros curriculares nacionais: Educação Física/Secretaria de Educação Fundamental. Brasília: MEC/SEF, 1997. 96p.

CASTEJÓN, F. J.; GIMÉNEZ, F. J. Teachers' perceptions of physical education content and influences on gender differences. Motriz, Rio Claro, v. 21 n. 4, p. 375-385, 2015.

CESCHINI, F. L.; ANDRADE, D. R.; OLIVEIRA, L. C.; ARAUJO JUNIOR, J. F.; MATSUDO, V. K. R. Prevalence of physical inactivity and associated factors among high school students from state's public schools. Jornal de Pediatria. Porto Alegre, v. 85, n. 4, 2009.

COLEDAM, D. H. C.; FERRAIOL, P. F.; JUNIOR, R. P.; DOS SANTOS, J. W.; OLIVEIRA, A. R. Factors associated with participation in sports and physical education among students from Londrina, Paraná State, Brazil. Cad. Saúde Pública. Rio de Janeiro, v. 30, n. 3, p. 533-545, 2014.

DAUENHAUER, B. D. e KEATING, X. D. The influence of physical education on physical activity levels of urban elementary students. Res. Q. Exerc. Sport. Estados Unidos, v. 82, p. 512-520, 2011.

DIAMANT, A. L.; BABEY, S. H.; WOLSTEIN, J. Adolescent Physical Education and Physical Activity in California. Copyright. UCLA Center for Health Policy Research, Estados Unidos, 2011. $8 \mathrm{p}$.

FARIAS JUNIOR, J. C.; LOPES, A. S.; MOTA, J. HALLAL, P. C. Prática de atividade física e fatores associados em adolescentes no Nordeste do Brasil. Rev. Saúde Pública. São Paulo, v. 46, n. 3 , p. 505-515, 2012.

FEITOSA, W. M. N.; TASSITANO, R. M.; TENÓRIO, M. C. M.; ALBUQUERQUE, A.; GUIMARÃES, F. J. P. S.; NETO, A. J. L. Aulas de educação física no ensino médio da rede pública estadual de caruaru: componente curricular obrigatório ou facultativo? R. da Educação Física/UEM, Matingá, v. 22, n. 1, p. 97-109, 2011.

GUERRA, P. H.; JÚNIOR, J. C. F.; FLORINDO, A. A. Comportamento sedentário em crianças e adolescentes brasileiros: revisão sistemática. Revista de Saúde Pública, São Paulo, v. 50, n. 9, p. 1-15, 2016. doi:10.1590/S1518-8787.2016050006307.

HALLAL, P. C.; KNUTH, A. G.; CRUZ, D. K. A.; MENDES, M. I.; MALTA, D. C. Prática de atividade física em adolescentes brasileiros. Ciência e Saúde Coletiva, Rio de Janeiro, v. 15, n. 2, p. 3035-3042, 2010.

JANSSEN, I. e LEBLANC, A. G. Systematic review of the health benefits of physical activity and fitness in school-aged children and youth. International Journal of Behavioral Nutrition and Physical Activity, Estados Unidos, v. 7, p. 40, p. 1-16. 2010. doi: 10.1186/14795868-7-40. 
KREMER, M. M.; REICHERT, F. F.; HALLAL, P. C. Intensidad y duración de los esfuerzos físicos en clases de Educación Física. Rev. Saúde Pública, São Paulo, v. 46, n. 2, 2012.

LAZZOLI, J. K.; NÓBREGA, A. C. L.; CARVALHO, T.; OLIVEIRA, M. A. B.; TEIXEIRA, J. A. C.; LEITÃO, M. B.; LEITE, N.; MEYER, F.; DRUMMOND, F. A.; PESSOA, M. S. V.; REZENDE, L.; ROSE, E. H.; BARBOSA, S. T.; MAGNI, J. R. T.; NAHAS, R. M.; MICHELS, G.; MATSUDO, V. Atividade física e saúde na infância e adolescência. Revista Brasileira de Medicina do Esporte, São Paulo, v. 4, n. 4, 1998.

NETO, A. R. M.; CRUZ, R. P.; SALGADO, S. S.; CHRISPINO, S. S.; SOARES, A. J. G. Evasão escolar e desinteresse dos alunos nas aulas de educação física. Pensar a Prática. Goiânia, v. 13, n. 2, p. 1-15, 2010.

NUNES, P.; PEREIRA, A.; ESPADA, M. A relação entre o género e motivação para a prática de atividades desportivas. Mediações - Revista OnLine da Escola Superior de Educação do Instituto Politécnico de Setúbal, Portugal, v. 3, n. 2, 2015.

OLIVEIRA, F.; MACEDO, R.; SILVA, A. Fatores associados a participação das alunas nas aulas de educação física: uma questão de gênero? Rev. Acta Brasileira do Movimento Humano, Paraná, v.4, n.5. p.73-86, 2014.

PAULA, T.; SILVA, J.; KOCIAN, R. C. A participação nas aulas de educação física escolar de ensino médio. Coleção Pesquisa em Educação Física, Várzea Paulista, v.10, n.6, 2011.

PALAKSHAPPA, D.; VIRUDACHALAM, S.; ORESKOVIC, N. M.; GOODMAN, E. Adolescent Physical Education Class Participation as a Predictor for Adult Physical Activity. Childhood Obesity, Estados Unidos, v. 11, n. 5, 2015. doi: 10.1089/chi.2015.0024.

PINHO, L.; BOTELHO, A. C. C.; CALDEIRA, A. P. Fatores associados ao excesso de peso em adolescentes de escolas públicas no norte de Minas Gerais. Revista Paulista de Pediatria, São Paulo, v. 32, n. 2, p. 237-43, 2014. doi: 10.1590/0103-0582201432216213.

REICHENHEIM, M. E.; MORAES, C. L. Pillars for assessing validity in epidemiological studies. Revista Brasileira de Epidemiologia. São Paulo, v. 1, n. 2, 1998.

REZENDE, L. F. M.; AZEREDO, C. M.; SILVA, K. S.; CLARO, R. M.; FRANÇAJUNIOR, I.; PERES, M. F. T.; LUIZ, O. C.; LEVY, R. B.; ELUF-NETO, J. The Role of School Environment in Physical Activity among Brazilian Adolescents. Plos One, v. 10, n. 6, e0131342. doi:10.1371/journal.pone.0131342. 2015.

SALLES-COSTA, R.; HEILBORN, M. L.; WERNECK, G. L.; FAERSTEIN, E.; LOPES, C. S. Gênero e prática de atividade física de lazer. Cad. Saúde Pública, Rio de Janeiro, v. 19, sup. 2, p. S325-S333, 2003.

SANTOS, S. J.; HARDMAN, C. M.; BARROS, S. S. H.; SANTOS, C. F. B. F.; BARROS, M. V. G. Association between physical activity, participation in Physical Education classes, and social isolation in adolescents. Jornal de Pediatria, Porto Alegre, v. 91, n. 6, p. 543-550, 2015. 
SILVA, D. A. S.; TREMBLAY, M. S.; PELEGRINI, A.; SILVA, J. M. F. L.; PETROSKI, E. L. Low aerobic fitness in Brazilian adolescents. Revista Brasileira de Medicina do Esporte. São Paulo, v. 21, n. 2, 2015.

SILVA, F. M. A.; SMITH-MENEZES, A. Participação em aulas de Educação Física e atitudes positivas para a prática de atividade física em adolescentes do estado de Sergipe, Brasil. Scientia Plena, Sergipe, v. 12, n. 8, 2016. doi: 10.14808/sci.plena.2016.082801.

SILVA, K. S.; NAHAS, M. V.; PERES, K. G.; LOPES, A. S. Factors associated with physical activity, sedentary behavior, and participation in physical education among high school students in Santa Catarina State, Brazil. Cad. Saúde Pública. Rio de Janeiro, v. 25, n. 10, p. 2187-2200, 2009.

SOUZA, J. C.; SOUZA, N.; ARASHIRO, E. S. H.; SCHAEDLER, R. Sonolência diurna excessiva em pré-vestibulandos. Jornal Brasileiro de Psiquiatria, Rio de Janeiro, v. 56, n. 3, p. 184-187, 2007.

TASSITANO, R. M.; BARROS, M. V. G.; TENÓRIO, M. C. M.; BEZERRA, J.; FLORINDO, A. A.; REIS, R. S. Enrollment in Physical Education Is Associated With HealthRelated Behavior Among High School Students. Journal of School Health, Estados Unidos, v. 80, n. $3,2010$.

TROST, S. G.; PATE, R. R.; SALLIS, J. F.; FREEDSON, P. S.; TAYLOR, W. C.; DOWDA, M.; SIRARD, J. Age and gender differences in objectively measured physical activity in youth. Medicine \& Science in Sports \& Exercise, Estados Unidos, v. 34, n. 2, 2002.

UCHOGA, L. A. R.; ALTMANN, H. Educação física escolar e relações de gênero: diferentes modos de participar e arriscar-se nos conteúdos de aula. Revista Brasileira de Ciências do Esporte. Brasília, v. 38, n. 2, p. 163-170, 2016.

Recebido em: 22/06/2018

Revisado em: 07/11/2018

Aprovado em: 18/03/2019

Endereço para correspondência:

barbaragicelia@hotmail.com

Bárbara Silva Araújo

Universidade Federal de Pernambuco

Av. Prof. Moraes Rego, 1235

Cidade Universitária,

50670-901 Recife - PE, Brasil. 